

Check for updates

\title{
fracturing dataset for benchmarking of enhanced geothermal system simulation tools
}

\begin{abstract}
Paromita Deb $\mathbb{D}^{1 凶}$, Stephan Düber $^{2}$, Carlo Guarnieri Calo' Carducci $\mathbb{C}^{3}$ \& Christoph Clauser $^{1}$
Successful design of enhanced geothermal systems (EGSs) requires accurate numerical simulation of hydraulic stimulation processes in the subsurface. To ensure correct prediction, the underlying model assumptions and constitutive relationships of simulators need to be verified against experimental datasets. With the aim of generating laboratory-scale benchmark datasets, a state-of-the-art testing facility was developed, allowing for experiments under controlled conditions. Samples of size $30 \mathrm{~cm} \times 30 \mathrm{~cm} \times 45 \mathrm{~cm}$ were subjected to confining stresses while high-pressure fluid was injected into the sample through a pre-drilled borehole, where a saw-cut notch was used to initiate a penny-shaped fracture. Fracture growth and propagation was monitored by measuring pressure data and acoustic emissions detected using 32 seismic sensors. Subsequently, samples were split along the fracture plane to outline the created fracture marked by a red-dyed injection fluid. Finally, a 2D fracture contour was generated using photogrammetry. Presented datasets, accessible via a public repository, include experiments on granite and marble samples. They can be used for verifying and improving numerical codes for field stimulation designs.
\end{abstract}

\section{Background \& Summary}

Engineering a successful stimulation system in the subsurface requires understanding rock response to different pressure conditions. The challenge is to predict as accurately as possible the growth and propagation of fractures in rocks during high-pressure fluid injection, a process called hydraulic stimulation. Several 2D and 3D tools developed by universities and industry are available to numerically simulate growth and predict fracture patterns for any geological condition. Due to the lack of analytical solutions for these coupled, non-linear processes, the accuracy of these simulation tools in solving hydro-mechanical processes can only be assessed by comparing the simulated results against laboratory-scale or field-scale datasets. A code comparison study investigating the capabilities of numerical codes and the quality of the numerical solutions to specific EGS problems is reported in $^{1}$, where real field data was used to benchmark a number of simulators under specific assumptions. Indeed, achieving a well-constrained field-scale hydraulic fracturing dataset is almost impossible due to the unknown geological complexity at depth, which requires major assumptions regarding boundary conditions and subsurface parameters. Therefore, conceivably well-controlled and repeatable laboratory-scale experiments are indispensable for verifying model assumptions and constitutive relationships used by numerical codes to support EGS design.

To obtain meaningful results from laboratory tests, it is necessary to perform experiments on sufficiently large samples so that a stable fracture propagation can be achieved. Experiments of this scale are rather expensive and relatively rare in the geothermal energy sector, especially when performed under true triaxial conditions, i.e. with confining stresses along all the three axes. A non-exhaustive list of true triaxial facilities available worldwide, which can accommodate large samples, is presented in Table 1 . However, most of these facilities are often commissioned by oil and gas companies, and therefore their results are not publicly available to the broad scientific community.

${ }^{1}$ Institute for Applied Geophysics and Geothermal Energy, RWTH Aachen University, Aachen, Germany. ${ }^{2}$ Chair of Geotechnical Engineering, RWTH Aachen University, Aachen, Germany. ${ }^{3}$ Institute for Automation of Complex Power Systems, RWTH Aachen University, Aachen, Germany. ${ }^{\bowtie e-m a i l: ~ p d e b @ e o n e r c . r w t h-a a c h e n . d e ~}$ 


\begin{tabular}{|c|c|c|c|c|c|}
\hline Institution & Country & Sample size $\left(\mathrm{mm}^{3}\right)$ & $\begin{array}{l}\text { Confining } \\
\text { Pressure }(\mathrm{MPa})\end{array}$ & \begin{tabular}{|l} 
Acoustic \\
Emission Data
\end{tabular} & Background \\
\hline $\begin{array}{l}\text { TerraTek Geomechanics Laboratory } \\
\text { (Schlumberger) }\end{array}$ & USA & $\begin{array}{l}762 \times 762 \times 914 \\
280 \times 280 \times 381\end{array}$ & $\begin{array}{l}55 \\
55 / 21\end{array}$ & Yes & Oil \\
\hline Halliburton & USA & $152 \times 152 \times 254$ & 17 & No & Oil \\
\hline Colorado School of Mines & USA & $300 \times 300 \times 300$ & 12 & Yes & Geothermal energy \\
\hline Earth Science Division, Berkeley & USA & $300 \times 300 \times 450$ & $14 / 7$ & Yes & Nuclear storage \\
\hline Beijing University of Petroleum & China & $300 \times 300 \times 300$ & 28 & No & Oil \\
\hline Jiangsu University of Mining & China & $500 \times 500 \times 500$ & 16 & Yes & Coal \\
\hline Jilin University & China & $300 \times 300 \times 300$ & 20 & yes & Geothermal energy, $\mathrm{CO} 2$ storage \\
\hline Tohoku University & Japan & $300 \times 300 \times 300$ & $>12$ & Yes & Stress measurements \\
\hline Yamaguchi University & Japan & $190 \times 190 \times 190$ & 6 & Yes & Geothermal energy, Oil \\
\hline Curtin University & Australia & $300 \times 300 \times 300$ & 50 & Yes & Oil \& Gas \\
\hline CSIRO Melbourne & Australia & $400 \times 400 \times 400$ & 25 & Yes & Oil \& Gas \\
\hline TU Delft & The Netherlands & $300 \times 300 \times 300$ & 39 & Yes & Oil \\
\hline Laboratoire de Mecanique de Lille & France & $500 \times 500 \times 500$ & 70 & Yes & Stress measurements \\
\hline
\end{tabular}

Table 1. Non-exhaustive list of comparable triaxial set-ups worldwide, list modified from ${ }^{3}$.

Against this background, a large-sample triaxial set-up was developed at RWTH Aachen University under a project funded by BMWi (Federal Ministry of Economics and Technology). The planning and construction of the testing facility, including the analytical and numerical validation of the final experimental procedure, is thoroughly discussed in ${ }^{2,3}$. The set-up allows for experiments on rocks with size $30 \mathrm{~cm} \times 30 \mathrm{~cm} \times 45 \mathrm{~cm}$ under defined and controlled boundary conditions, yielding reproducible and repeatable datasets, well-suited for code benchmarking.

In this paper we present hydraulic stimulation datasets from experiments where the borehole axis was parallel to the minimum horizontal stress direction and therefore the plane of crack propagation was parallel to the maximum horizontal stress direction. Investigated rock samples represent the reservoir rock types of a potential EGS site in Mexico. The datasets represent hydraulic stimulation responses in quasi-homogenous and extremely heterogeneous crystalline rock types, such as, a very fine-grained granite and a coarse-grained marble, respectively. Additionally, a dense network of 32 acoustic sensors, comprising of $28 \mathrm{GmuG}$ standard ultrasonic sensors (http://www.gmugmbh.de/prod.html) and 4 Glaser amplitude-calibrated sensors ${ }^{4,5}$, was used to track the fracture propagation in real time.

The validation dataset includes fluid injection rate, pressure in the injection interval, confining stresses, mechanical and petrophysical properties of the rock specimens, properties of the injection fluid, mechanical details of the experimental set-up, and acoustic emission data. Additionally, we provide a Python-based code which can be used for processing the seismic data and visualizing of the experimental results.

\section{Methods}

The main components of the experimental set-up, sample requirements and experimental protocol are described separately in the following sub-sections.

Sample. Experiments were performed on dense, very low-porosity and low-permeability blocks of granite and marble, cut and polished to final dimensions of $30 \mathrm{~cm} \times 30 \mathrm{~cm} \times 45 \mathrm{~cm}$. A borehole of radius $10 \mathrm{~mm}$ was drilled through the horizontal center of the rock, where a circumferential notch with radius $17 \pm 1 \mathrm{~mm}$ was cut into the borehole wall at a height of $\mathrm{z}=22.5 \mathrm{~cm}$ to guide the crack initiation.

The tool for cutting the notch, shown in Fig. 1, was developed in-house and described in $^{2}$. It is made of a diamond cutting disc centrally mounted on a long drive shaft. A rotary motor is connected to one end of the shaft, while the other end is mounted in a handle. To cut the notch, the tool is placed centrally in the bore hole of the sample. Then the notch is initially cut in one direction to full depth until the shaft touches the borehole wall. Subsequently, the cutting tool is progressively moved around to create a circular notch on the borehole wall. A guiding aid was used to ensure that the notch depth is $7 \pm 1 \mathrm{~mm}$ all around.

The six surfaces of the samples were alphabetically numbered from A to F. A Cartesian coordinate system was used to systematically describe the samples. The origin of the coordinate system is located in the center of the surface A, the top of the sample. The $\mathrm{z}$-direction corresponds to the borehole axis and points from the upper side A towards the lower side $\mathrm{F}$. The $\mathrm{y}$-axis points from surface $\mathrm{E}$ to $\mathrm{C}$ and the $\mathrm{x}$-axis is positive from surface $\mathrm{B}$ to $\mathrm{D}$ (Fig. 1).

Experimental set-up. The main components of the triaxial set-up for the hydraulic stimulation test are the loading, injection and acoustic data acquisition systems.

Loading system. The experiments were performed with samples under three-dimensional confining stress generated by a massive iron loading frame $(4600 \mathrm{~kg})$ supported by stiff abutments. Desired stress conditions were achieved by pressurizing metal flat-jacks interleaved between the loading frame and thick iron loading plates leaning against the faces of the sample (Fig. 2). In order to account for the flat-jack rigidity at the edges, their sizes exceed the loading plate dimensions at each side by $10 \mathrm{~mm}$ (A, F: $320 \times 320 \mathrm{~mm}$; B, C, D and E: $320 \times 470 \mathrm{~mm}$ ), 


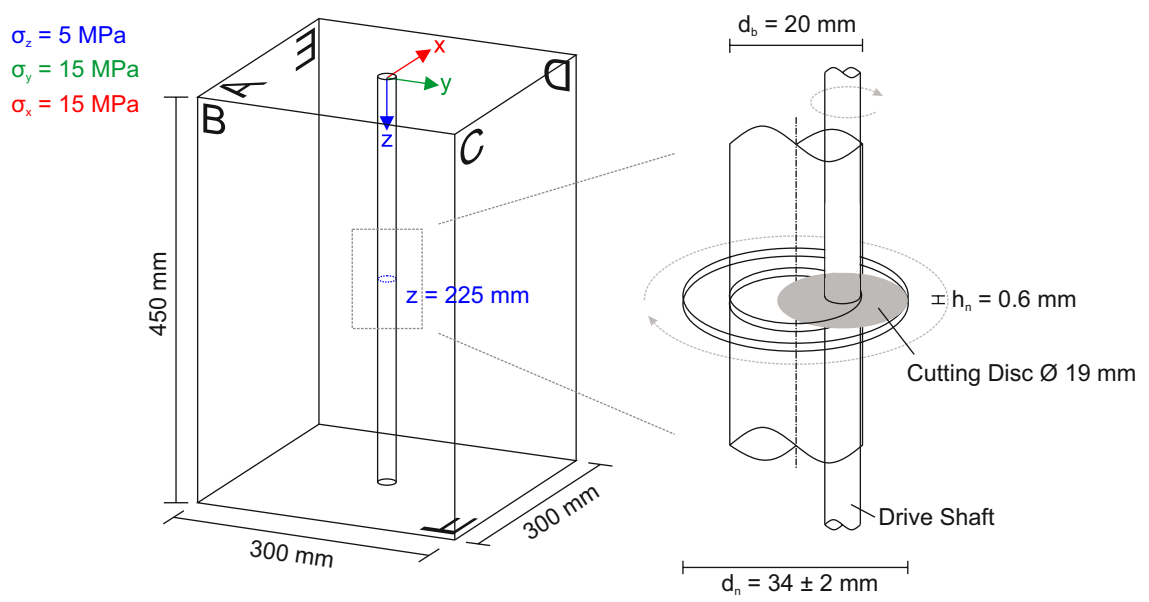

Fig. 1 Geometry of the sample, borehole and the notch: naming of sample surfaces (A-F), coordinate system and depth of the notch ( $\mathrm{z}$ (left); arrangement for creating a notch in the borehole using cutting disc (right), $\mathrm{d}_{\mathrm{n}}$ and $h_{n}$ are the diameter and thickness of the notch respectively, $d_{b}$ is the diameter of the borehole. .

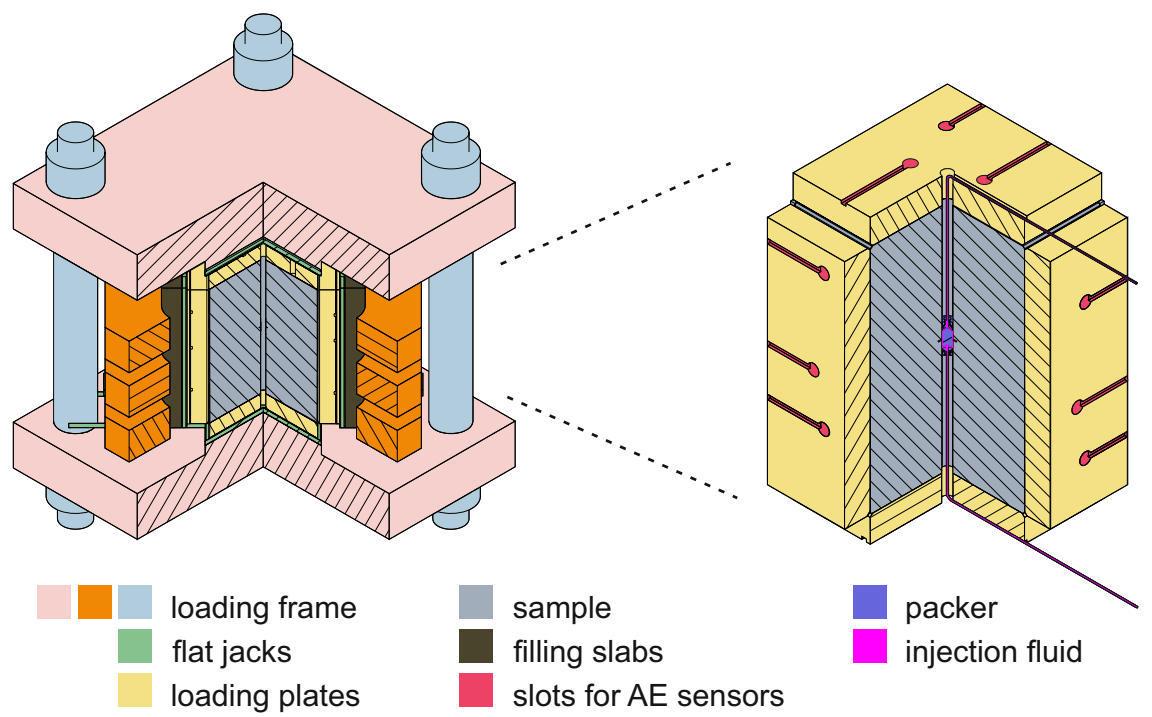

Fig. 2 Schematic figure of the triaxial set-up: components of the loading system (left); details of the slots for the acoustic sensors in the loading plates and a cross-section through the sample showing the borehole and the packer configuration (right).

ensuring that the whole area of the sample is uniformly loaded. Teflon films with thickness $1 \mathrm{~mm}$ were inserted between each sample face and relative loading plate to reduce the iron-to-rock friction. The edges of the load plates facing the sample were also chamfered to avoid contact between contiguous elements. Circular slots were drilled in the loading plates for accommodating the acoustic sensors, thus ensuring a direct contact with the sample.

Injection system. The injection system is composed of a pump, pipes, pressure sensors, a packer and injection fluid. In the center of the $45 \mathrm{~cm}$ long borehole, a packer was used to isolate an open-hole section of $30 \mathrm{~mm}$ that defines the injection interval (Fig. 3). The packer is terminated by threaded washers that once tightened, press the O-ring against the borehole wall sealing the injection interval from the rest of the borehole and fixing the packer in its position. Measurements were performed in separate experiments, which also allowed for the determination of the bulk modulus (compressibility) of both injection system and pump. Table 2 summarizes the different parameters of the injection system.

The liquid tank valve A and the bleeding line valve C (Fig. 3) were closed during the experiment and the volume of the pump cylinder is reduced to pressurize the injection system. The pressure was measured using two pressure transmitters $p 1$ and $p 2$ located at the upstream and downstream sections of the injection interval. In order to account for fluid viscosity and improve the measurement accuracy, the pressure of the injection interval was assumed to be the average of $p 1$ and $p 2$. 


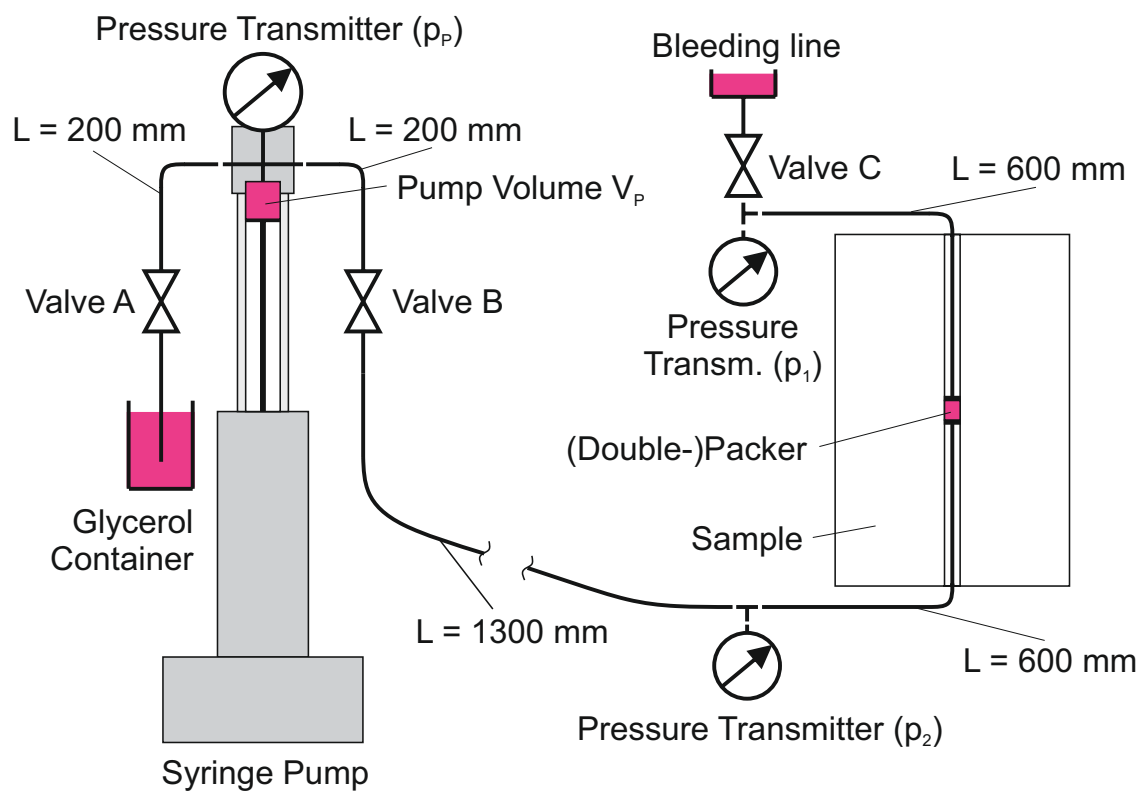

Fig. 3 Schematic figure of the injection system: details of valves, sensors and pump position in the hydraulic circuit.

\begin{tabular}{|l|l|}
\hline Components & Specifics \\
\hline Pump & Syringe pump, Teledyne Isco 260 HP \\
\hline Pressure transmitters & Keller P33x/1000 bar/80794 \\
\hline Inner diameter of the pipes & $1.76 \mathrm{~mm}$ \\
\hline Volume of the injection system & $12 \mathrm{~cm}^{3}$ \\
\hline Initial pump volume & $5 \mathrm{~cm}^{3}$ \\
\hline Height of the open hole section & $30 \mathrm{~mm}$ \\
\hline Radius the borehole & $10 \mathrm{~mm}$ \\
\hline Radius of radial notch & $17 \pm 1 \mathrm{~mm}$ \\
\hline Thickness of radial notch & $0.6 \mathrm{~mm}$ \\
\hline Bulk modulus of pump & $0.18 \mathrm{GPa}$ \\
\hline $\begin{array}{l}\text { Bulk modulus of injection system (pipes, } \\
\text { pressure transmitters and packer) }\end{array}$ & $2.7 \mathrm{GPa}$ \\
\hline
\end{tabular}

Table 2. Specifics of the injection system.

The injection fluid used in the experiment is a mixture of $98 \%$ glycerol and $2 \%$ ink. The red ink was added to the injection fluid to mark the propagation of the fracture and measure the fracture radius after the sample is split along the fracture plane. The dynamic viscosity of the injection fluid is highly temperature-dependent and therefore characterized in a separate test for the temperatures of the experiments using a rotary rheometer. The viscosity values of the injection fluid were then derived for each experiment from the temperature recorded during the experiment. The air content in the injection system was estimated based on the method described in ${ }^{6}$.

Acoustic data acquisition system. The acoustic data are acquired using the GMuG data acquisition system (http://www.gmugmbh.de/prod.html), which can operate both in active transmission (AT) and acoustic emission (AE) mode, i.e. in response to a pressure impulse generated actively and seismicity induced due to injection, respectively. The system comprises of piezoelectric sensors, pre-amplifiers, a low-noise multi-channel amplifier and a high-voltage amplifier for pressure impulse generation. The piezoelectric element has a circular contacting point with a diameter of $8 \mathrm{~mm}$, and operates in the bandwidth from $20 \mathrm{kHz}$ to $1 \mathrm{MHz}$ both as transmitter and receiver. Each sensor, with outer body diameter $20 \mathrm{~mm}$, has a defined allotted slot in the loading plates, where a tuneable spring is used to generate a constant and repeatable pressure between the specimen and the sensor. A total of 32 acoustic sensors are distributed over the surface of the sample in non-redundant positions for maximum azimuthal coverage. The original configuration was modified replacing the GMuG-type transducers in the channels $(9,15,20$ and 30) with four Glaser-type conical piezo-electric sensors, laboratory-standard displacement sensors characterized by a quasi-flat wide-band response between $50 \mathrm{kHz}$ and $1 \mathrm{MHz}^{4}$. 


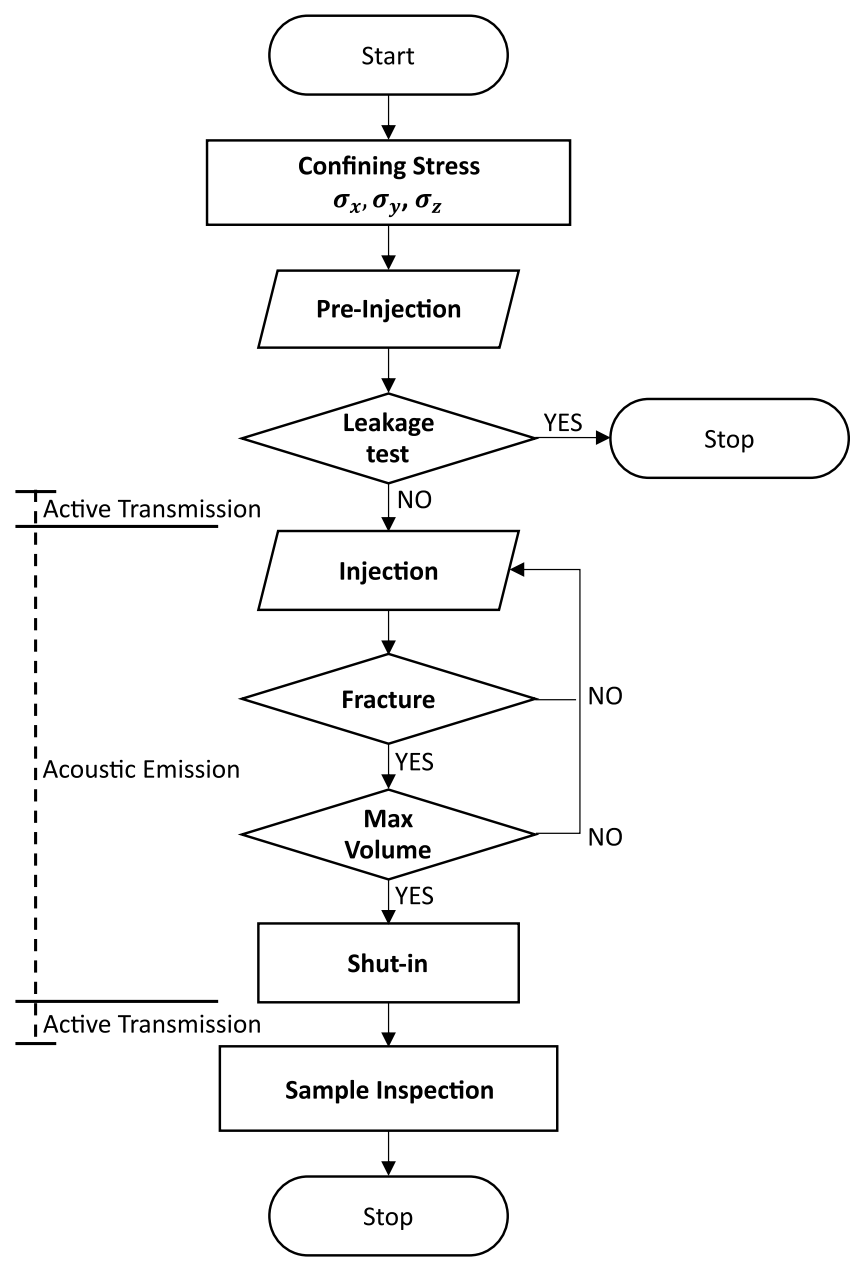

Fig. 4 Experimental protocol steps: simplified flow diagram of the protocol explained in the Methods section.

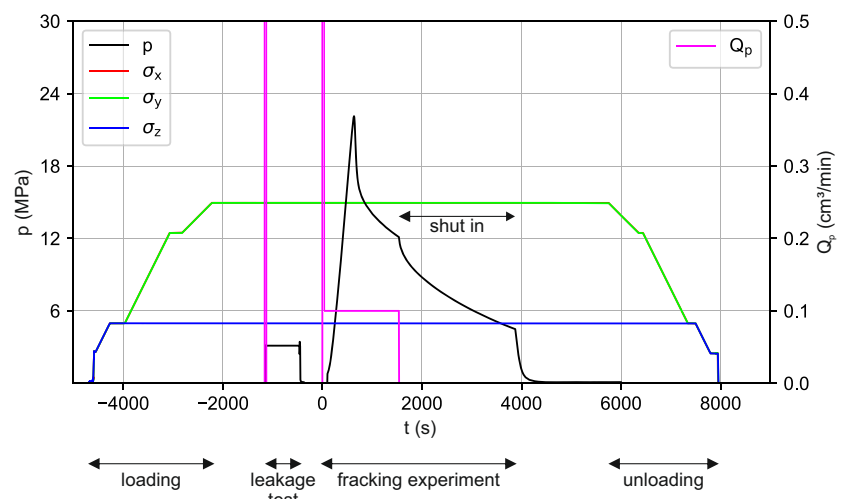

Fig. 5 Experiment stages: applied confining stresses $\sigma_{x}, \sigma_{y}$, and $\sigma_{z}(\mathrm{MPa})$, pressure $\mathrm{p}(\mathrm{MPa})$ and flow rate $\mathrm{Q}_{\mathrm{p}}$ $\left(\mathrm{cm}^{3} / \mathrm{min}\right)$.

The control unit consists of a dedicated PC equipped with four M2i3122 12-bit 8-channel simultaneous sampling analog-to-digital (ADC) cards and one M2i6011 14-bit digital-to-analog (DAC) card synchronized via M2i Star-Hub technology. The data acquisition was controlled via GMuG software, which allows the configuration of each sensor channel setting and stores the acquired data in binary format both in the standard SEG-Y and in a proprietary $\mathrm{GMuG}$ format.

The data collected in AT mode were used for determining average P-wave velocity in the sample. Experiments were performed by stimulating the sample using one sensor at a time as a transmitter, while all the other sensors acted as receivers, generating a total of 868 records: 28 transmitters (excluding Glaser sensors) times 31 acoustic receivers. The transmitter generates a pressure pulse which is obtained by the modulation of a carrier signal at 

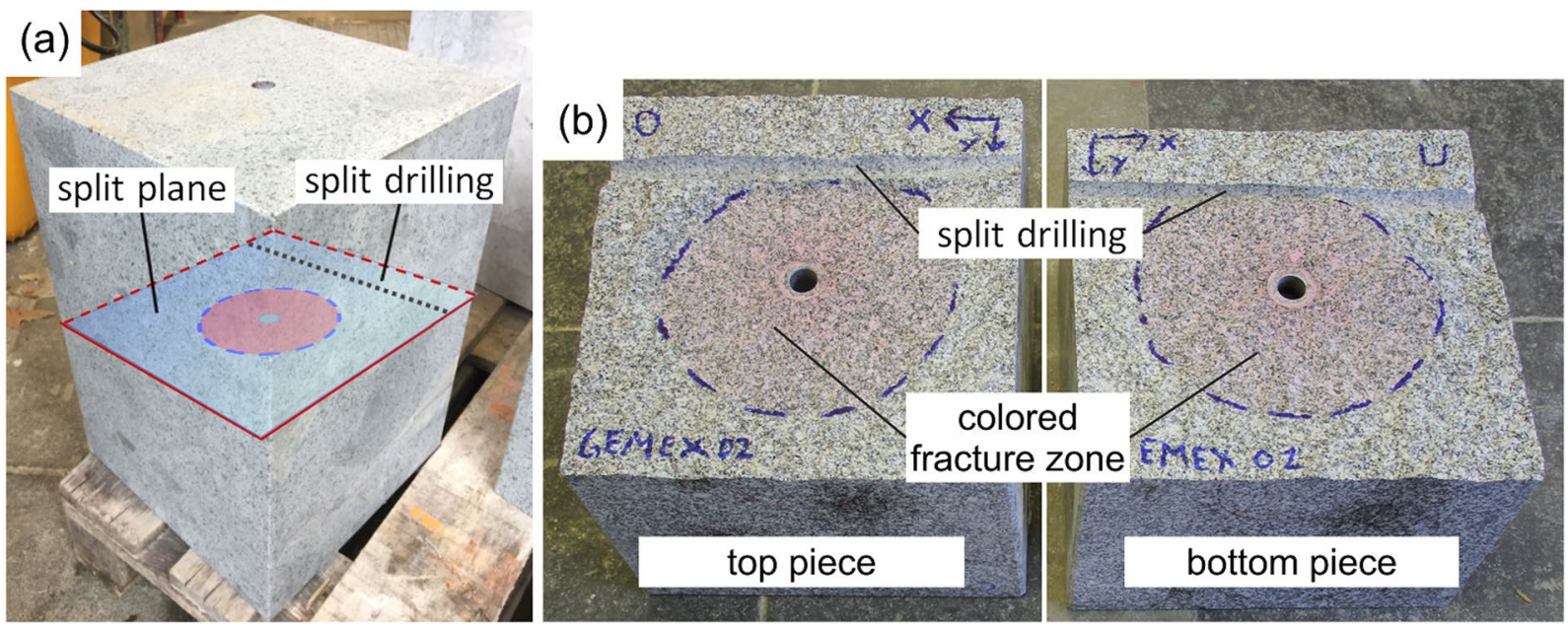

Fig. 6 Visual inspection of created fracture: splitting of the sample along fracture plane (a), demarcation of the fracture diameter based on the extent of spread of the colored ink $(\mathbf{b})$.

\begin{tabular}{|l|l|}
\hline $\begin{array}{l}\text { Contents of Data } \\
\text { Repository }\end{array}$ & Details \\
\hline AE & scripts for elaborating acoustic emission data \\
\hline AT & scripts for elaborating active transmission data \\
\hline DOC & detailed documentation and examples for running the processing code \\
\hline GEMex02 & experimental Data of GEMex02 \\
\hline GEMex03 & experimental Data of GEMex03 \\
\hline GEMex04 & experimental Data of GEMex04 \\
\hline GEMex06 & experimental Data of GEMex06 \\
\hline SystemProperties & experimental boundary conditions and injection system \\
\hline
\end{tabular}

Table 3. Data Repository structure.

$1 \mathrm{MHz}$ with $\cos ^{4}$ envelope function. The P-wave arrival time $t^{p}$ was then calculated using the Akaike Information Criterion (AIC) ${ }^{7}$ over the time series $y(n)$ of length $\mathrm{N}$ and sampling period $\Delta t$ as

$$
\begin{gathered}
t^{p}=\Delta t \cdot \underset{k}{\arg \min _{k}} \operatorname{AIC}(k), \text { with } \\
\operatorname{AIC}(k)=k \cdot \log (\operatorname{var}(y(1: k)))+(\mathrm{N}-k-1) \cdot \log (\operatorname{var}(y(k+1: \mathrm{N}))) .
\end{gathered}
$$

Once the first arrivals are picked for every transmitter-receiver pair, their distance was plotted against the $\mathrm{P}$-wave arrival time. A linear regression of all the events provided the average $\mathrm{P}$-wave velocity in the sample which is the value that minimises the sum of squared errors. Signals corresponding to transmitter-receiver pairs which were on the same side of the sample were discarded in the linear regression to avoid false picks related to surface waves.

The data collected in AE mode were used for determining the position of the acoustic source induced during the fracturing of the sample. When a signal on any of the channels exceeds a defined voltage threshold, it triggers the synchronized acquisition in all of the 32 sensors. The signals were sampled at a rate of $10 \mathrm{MHz}$ over a time window of 4096 samples equivalent to $410 \mu$ s, with a pre-trigger interval equal to $30 \%$ of the window width. For a given acoustic event, the first arrival $t_{i}^{p}$ for a sensor $i$ was picked using the same AIC criterion as in the case of active transmission experiments. Since the localisation of the seismic events requires the minimisation of a four-dimensional error function $e(x, y, z, t)$, at least four sensors must detect the event. If this condition is satisfied, then the position of the event $\left(x_{E}, y_{\mathrm{E}}, \mathrm{z}_{E}\right)$ and the actual event time $t_{0}$ is obtained from the information on the coordinates of the sensors $\left(x_{\mathrm{i}}, y_{i}, z_{i}\right)$, the measured first arrival $t_{i}^{p}$ and the estimated P-wave propagation speed $v$ in the specimen.

$$
\begin{gathered}
e(x, y, z, t)=\sum_{N_{C h}}\left|\left(\frac{\sqrt{\left(x_{i}-x_{E}\right)^{2}+\left(y_{i}-y_{E}\right)^{2}+\left(z_{i}-z_{E}\right)^{2}}}{v}+t_{0}\right)-t_{i}^{p}\right|^{2} \\
{\left[x_{E}, y_{E}, z_{E}, t_{0}\right]=\arg \min _{(x, y, z, t)}(e)}
\end{gathered}
$$




\begin{tabular}{|l|l|l|}
\hline Name of the file & Format & Detail \\
\hline GEMex02_InjectionData & .txt & pressure, volume and flow-rate versus absolute time \\
\hline GEMex02_SensorPositions & .txt & position of acoustic sensors on the surface of the sample \\
\hline GEMex02_NullTime & .txt & reference time for time conversion from absolute time to time of injection start \\
\hline GEMex02_RockProperties & .txt & petrophysical and mechanical properties \\
\hline GEMex02_Fluidproperties & .txt & Injection fluid properties \\
\hline GEMex02_FractureRadii & .txt & fracture radii obtained from photogrammetry \\
\hline 01-AT_Before_HF & folder & $\begin{array}{l}\text { active transmission data acquired before the hydraulic fracturing experiment } \\
\text { with sample under confining stresses }\end{array}$ \\
\hline 02-AE_During_HF & folder & $\begin{array}{l}\text { acoustic emission acquired during the hydraulic fracturing experiment with } \\
\text { sample under confining stresses }\end{array}$ \\
\hline 03-AT_After_HF & folder & $\begin{array}{l}\text { active transmission data acquired after the hydraulic fracturing experiment } \\
\text { with sample under confining stresses }\end{array}$ \\
\hline GEMex02-F & .png & image showing the fracture radius of the split sample \\
\hline
\end{tabular}

Table 4. Example of the data content of a particular experimental folder (GEMex02).

The $\arg \min (e)$ was obtained using the fminsearch method, which allows the minimisation of a multi-dimensional function through the derivative free Nelder-Mead simplex method ${ }^{8}$.

Experimental protocol. Details of the experimental protocol steps are summarised below, whereas the salient stages are graphically depicted in Fig. 4. Example of a pressure response curve versus injection rate during different stages of an experiment is shown in Fig. 5.

a. The confining stresses $\left(\sigma_{x}=\sigma_{y}=15 \mathrm{MPa}, \sigma_{z}=5 \mathrm{MPa}\right)$ are applied on the sample.

b. All the valves A, B and C are opened and the injection fluid is circulated through the system (from the glycerol container to the bleeding line) to de-air the pipelines and saturate the injection interval (Fig. 3).

c. A leakage test is carried out to assess the tightness of the injection system. Valves A and C are closed and the injection system is pressurized to $3 \mathrm{MPa}$ for approximately $600 \mathrm{~s}$ (Fig. 5). The required fluid volume which has to be injected into the borehole to maintain constant pressure is determined and used as an indicator of the tightness of the system.

d. The active transmission experiment is performed to determine the average P-wave velocity of the sample before the fracturing event.

e. The fluid volume $V_{0}$ stored in the pump is reduced to $5 \mathrm{~cm}^{3}$ and the injection of fluid into the sample starts. This time instant is referred to as null time $(t=0 \mathrm{~s})$ for all recorded data. Simultaneously, the acoustic data acquisition system is configured in acoustic emission mode to begin acquisition of induced seismic events.

f. As injection continues, the pressure in the fluid system builds up linearly until the fluid-pressure overcomes the minimum principal stress and the tensile strength of the rock, and the fracture initiates ${ }^{9}$. From this point onwards, the pressure increase until the peak pressure (also called breakdown pressure) is a function of the viscosity of the injection fluid and is monitored using the pressure rate.

g. Once the breakdown pressure is reached, only a pre-defined fluid volume increment $\Delta V_{p}$ is further injected into the system to curtail the fracture growth. Afterwards the pump is stopped and the experiment enters the "shut-in" phase.

h. During shut-in, the pressure decreases gradually until it falls below the minimum confining stress ( $\sigma_{z}=5 \mathrm{MPa}$ ), following which the pressure in the injection interval is released.

i. The acoustic emission recording is stopped and an active transmission experiment is repeated to evaluate the change in $\mathrm{P}$-wave velocity after fracture formation and propagation.

j. The loading frame is disassembled and the sample is removed from the experimental set-up to be split along the fracture plane by means of a second hole drilled perpendicular to the original borehole (Fig. 6(a)).

$\mathrm{k}$. The extent of the fracture affected zone is demarcated by the spread of the red dye used in the injection fluid and the fracture radius is measured (Fig. 6(b)).

\section{Data Records}

All experimental data are published via Zenodo Repository ${ }^{10}$. The data repository structure is shown in Table 3 . The data structure within each experimental folder is presented in Table 4.

The acoustic emission data are stored in three sub-folders for each experiment. Two folders contain data from active transmission experiments conducted before and after fracturing while the sample is under confining stress, and the third one consists of induced seismicity data recorded during fracturing. Within each of these folders, seismic data are provided in binary format both in GmuG format and SEGY standard format.

\section{Technical Validation}

The engineering details and the experimental system validation are discussed in details in $^{2,3}$. The final adopted experimental procedure is the result of numerous preliminary experiments, which established the validity and repeatability of the measurements ${ }^{2,3}$. 

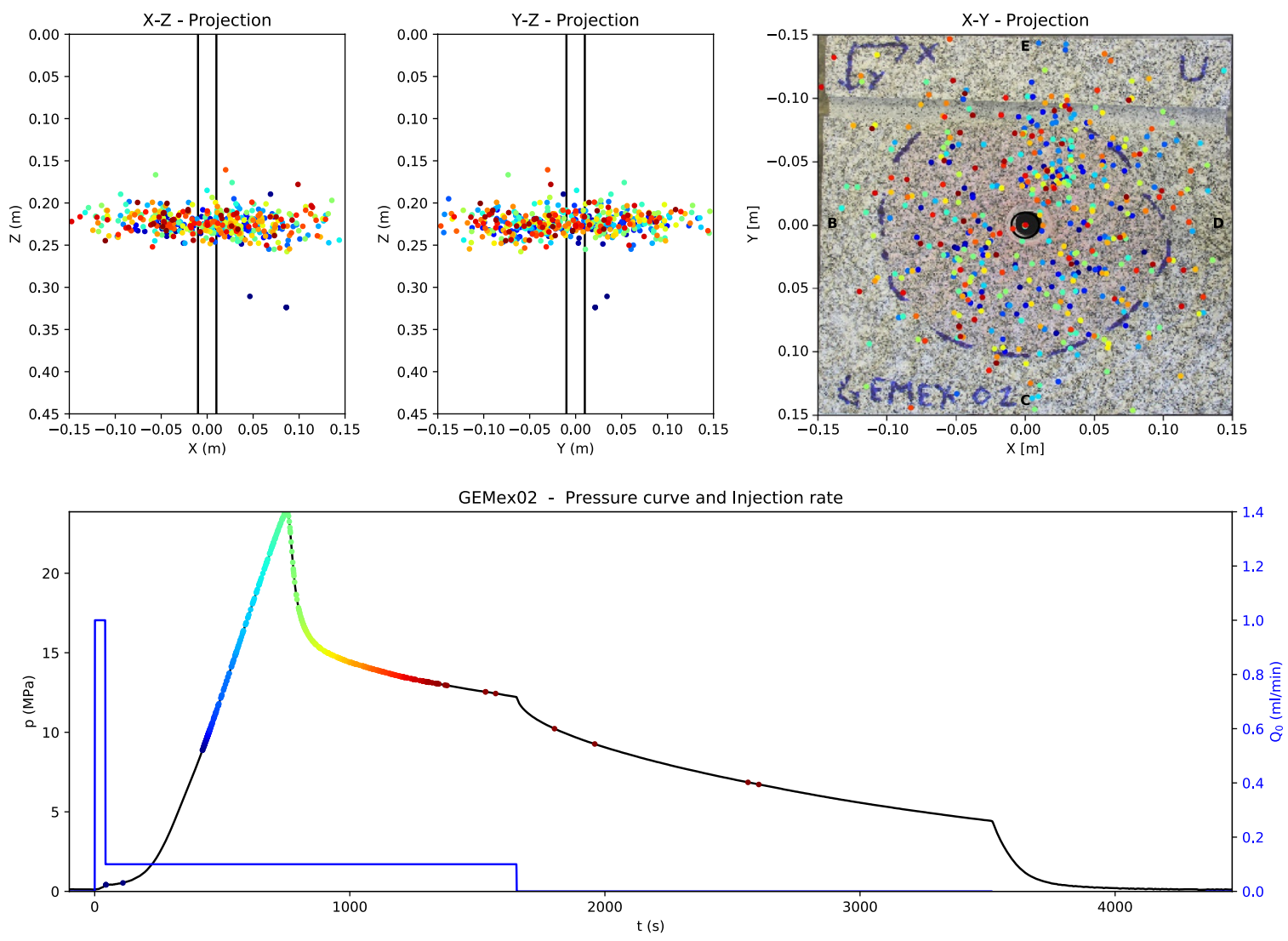

Fig. 7 Processed output of a hydraulic fracturing experiment: Final outcome from the processing of GEMex 02 using the provided Python code; localised acoustic emission events in X-Z (top-left) and Y-Z (top-center) projections; photograph of the specimen split along the fracture plane with superimposed localised events and outline (blue) of the fracture (top-right); pressure and injection rate versus time and acoustic emission events with time proportional color scale (bottom).

Results obtained from the processing of one of the experiments performed in a granite specimen (GEMex 02), are reported in Fig. 7. The expected stages of the fracturing process can be identified in the pressure profile: pressure rise, breakdown pressure, fracture propagation, and, finally, pressure decay. Pressure increases linearly with the applied constant injection rate to a point called fracture-initiation pressure, where the trend becomes non- linear. The exact time position of this point in the pressure curve cannot be directly identified by visual inspection, but needs to be calculated from the pressure rate curve $(d p / d t)$. Further increase of pressure, until the breakdown pressure $p_{b}$, is theoretically a function of the injection fluid viscosity and pressurization rate. In most of our experiments, the fracture initiation pressure is equivalent to the breakdown pressure, after which the pressure decays rapidly. The growth of the fracture is limited by injecting only a limited volume of fluid.

Acquired data, besides being qualitatively consistent with theoretical pressure responses ${ }^{11}$, are also characterized by the accuracy of the measuring instruments, $0.05 \mathrm{MPa}$ for the pressure and $0.5 \%$ of the reading for the flow rate, respectively, and a fluid volume step resolution of $16.6 \times 10^{-6} \mathrm{ml}$.

In addition to the pressure profile, the localized acoustic emission events processed using the code provided are also plotted in Fig. 7. The exact localization of events depends strongly on the specific algorithm used for seismic data processing. Although simple in the implementation, this method is very sensitive to errors in the $\mathrm{P}$-wave time of arrival, which are reflected in a significant uncertainty in the AE source localization. More robust techniques should be incorporated to improve the localization accuracy. However, the processed results using the basic code provided within the repository already correlate well with the spread of the dyed injection fluid which delineates the fracture-affected zone (Fig. 7).

\section{Usage Notes}

An example of code execution is provided in the DOC folder (see Table 3) together with flow-diagrams for the most complex scripts as a short documentation. For best compatibility of the Python code, the use of Microsoft Windows environment is recommended.

\section{Code availability}

Python scripts for basic processing of seismic data and aggregate visualization of both acoustic events and pressure profiles are published in the data repository. Requirements for scripts execution are Python 2.7 and the additional modules numpy and matplotlib. The code in the repository relies on methods widely available in literature, and is therefore provided "as-is", with no other warranties, expressed or implied, of correctness and 
completeness. It is not to be considered as a software for professional data elaboration but rather as an example in support of the research community to develop specific codes by providing a starting template. Users are encouraged to view the code and make necessary changes as required.

Received: 20 March 2020; Accepted: 4 June 2020;

Published online: 08 July 2020

\section{References}

1. White, M.D. et al. Benchmark Problems of the Geothermal Technologies Office Code Comparision Study. Report No. PNNL-26016 (U.S. Department of Energy, Washington, 2016).

2. Siebert, P. Laborversuche zur hydraulischen Risserzeugung in dreiaxial belasteten Granitquadern http://publications.rwth-aachen.de/ record/716212 (2017).

3. Clauser, C., Willbrand, K., Ziegler, M., Feinendegen, M., Siebert, P., Fries, T.P., Weber, N. Entwicklung eines Werkzeugs zur Auslegung von HDR-Risssystemen, BMWi-Projekt 0325167 (RWTH Aachen University, Aachen, 2015).

4. KRN Services, Inc., http://www.krnservices.com/documents/bbpc_flyer_4-13-15.pdf (2015)

5. McLaskey, G. C. \& Glaser, S. D. Acoustic Emission Sensor Calibration for Absolute Source Measurements. J. Nondestruct Eval. 31, $157-168(2012)$

6. Kim, S. \& Murrenhoff, H. Measurement of effective bulk modulus for hydraulic oil at low pressure. Journal of Fluids Engineering. 134, 2 (2012)

7. Akaike, H. Information theory and an Extension of the Maximum Likelihood Principle. In: Parzen E., Tanabe K., Kitagawa G. (eds). Selected Papers of Hirotugu Akaike. Springer Series in Statistics, 199-213 (Springer, New York, 1998).

8. Lagarias, J. C., Reeds, J. A., Wright, M. H. \& Wright, P. E. Convergence Properties of the Nelder-Mead Simplex Method in Low Dimensions. SIAM Journal of Optimization. 9, 112-147 (1998).

9. Hubbert, M. K. \& Willis, D. G. Mechanics of Hydraulic Fracturing. Transactions of Society of Petroleum Engineers of AIME 210, 153-163 (1957)

10. Deb, P., Düber, S., Guarnieri Calo' Carducci, C. \& Clauser, C. Dataset related to the article "Laboratory-scale hydraulic fracturing dataset for benchmarking of Enhanced Geothermal System simulation tools". Zenodo https://doi.org/10.5281/zenodo.3710746 (2020).

11. Zoback, M.D. Determination of $\mathrm{S}_{3}$ from mini-fracs. In: Reservoir Geomechanics (Cambridge, New York, 2007).

\section{Acknowledgements}

We thank Dr. Philipp Siebert for his previous work at GIB (Chair of Geotechnical Engineering, RWTH Aachen University) in designing, developing and characterizing the experimental set-up and Dr. Karen Willbrand for her past work at GGE (Institute for Applied Geophysics and Geothermal Energy) in developing the data processing algorithms. This project is funded by European Union's H2020 project GEMex with Grant Agreement No 727550.

\section{Author contributions}

Paromita Deb coordinated the experiments, performed the measurements of rock petrophysical properties, collected the acoustic emission data and drafted the manuscript. Stephan Düber took part in the collection of samples from Mexico, collected and processed the mechanical data. Paromita Deb and Stephan Düber directed and supervised the hydraulic fracturing experiments. Carlo Guarnieri Calo' Carducci contributed to improve the acoustic emission acquisition system. Christoph Clauser conceived the original project idea, acquired the funding and provided feedback and scientific advice throughout the project. All authors contributed to writing and reviewing the final manuscript.

\section{Competing interests}

The authors declare no competing interests.

\section{Additional information}

Correspondence and requests for materials should be addressed to P.D.

Reprints and permissions information is available at www.nature.com/reprints.

Publisher's note Springer Nature remains neutral with regard to jurisdictional claims in published maps and institutional affiliations.

(c) (i) Open Access This article is licensed under a Creative Commons Attribution 4.0 International cc. License, which permits use, sharing, adaptation, distribution and reproduction in any medium or format, as long as you give appropriate credit to the original author(s) and the source, provide a link to the Creative Commons license, and indicate if changes were made. The images or other third party material in this article are included in the article's Creative Commons license, unless indicated otherwise in a credit line to the material. If material is not included in the article's Creative Commons license and your intended use is not permitted by statutory regulation or exceeds the permitted use, you will need to obtain permission directly from the copyright holder. To view a copy of this license, visit http://creativecommons.org/licenses/by/4.0/.

The Creative Commons Public Domain Dedication waiver http://creativecommons.org/publicdomain/zero/1.0/ applies to the metadata files associated with this article.

(c) The Author(s) 2020 